\title{
Damsels in distress - Seasons, habitat structure and water pollution changes damselfly diversity and assemblage in urban wetlands
}

\begin{abstract}
Pankaj Koparde ${ }^{1,2}$
107, Radhika Apartment, Survey no. 12/1/2, Anandnagar, Sinhgad road, Pune-411051, Maharashtra, India ${ }^{2}$ Current affiliation: Sálim Ali Centre for Ornithology \& Natural History, Anaikatty (Post), Mungilpallam, Coimbatore - 641108, Tamil Nadu, India
\end{abstract}

\section{Supplementary Material}

Appendix S1. List of odonates observed at studied localities. Numbers in the brackets show number of species in the given family. Abbreviation and symbol: X, presence; *, recorded off transect.

\begin{tabular}{|c|l|c|c|c|c|c|c|}
\hline No. & Scientific Name & KDK & PL & PLD & SNT & VLH & VSU \\
\hline $\begin{array}{c}\text { Suborder: Anisoptera } \\
\text { Family: Aeshnidae (4) }\end{array}$ & & & & & & \\
\hline 1 & Anax spp. & & & & & & $\mathrm{X}$ \\
\hline 2 & Anax guttatus (Burmeister, 1839) & $\mathrm{X}$ & $\mathrm{X}$ & & & & $\mathrm{X}$ \\
\hline 3 & Anax immaculifrons (Rambur, 1842) & & & & & & $\mathrm{X}$ \\
\hline 4 & Gynacantha bayadera* Selys,1891 & & & & $\mathrm{X}$ & & \\
\hline Family: Gomphidae (3) & & & & & & \\
\hline 5 & Burmagomphus spp. & & & & & & $\mathrm{X}$ \\
\hline 6 & Ictinogomphus rapax (Rambur, 1842) & $\mathrm{X}$ & $\mathrm{X}$ & & $\mathrm{X}$ & $\mathrm{X}$ & $\mathrm{X}$ \\
\hline 7 & Microgomphus annulatus* (Selys, 1854) & & & & & & $\mathrm{X}$ \\
\hline Family: Libelullidae (20) & & & & & & \\
\hline 8 & Acisoma panorpoides Rambur, 1842 & & $\mathrm{X}$ & & & & $\mathrm{X}$ \\
\hline 9 & $\begin{array}{l}\text { Brachythemis contaminata } \\
\text { (Fabricius, } 1793 \text { ) }\end{array}$ & $\mathrm{X}$ & $\mathrm{X}$ & & $\mathrm{X}$ & $\mathrm{X}$ & $\mathrm{X}$ \\
\hline 10 & Bradinopyga geminata (Rambur, 1842) & & & & $\mathrm{X}$ & & $\mathrm{X}$ \\
\hline 11 & Crocothemis servilia (Drury, 1770) & $\mathrm{X}$ & $\mathrm{X}$ & & $\mathrm{X}$ & $\mathrm{X}$ & $\mathrm{X}$ \\
\hline 12 & Diplacodes trivialis (Rambur,1842) & $\mathrm{X}$ & $\mathrm{X}$ & & $\mathrm{X}$ & $\mathrm{X}$ & $\mathrm{X}$ \\
\hline 13 & Neurothemis fulvia (Drury, 1773) & $\mathrm{X}$ & & & & $\mathrm{X}$ & \\
\hline 14 & Orthetrum sabina (Drury, 1773) & $\mathrm{X}$ & $\mathrm{X}$ & & $\mathrm{X}$ & & $\mathrm{X}$ \\
\hline
\end{tabular}




\begin{tabular}{|c|c|c|c|c|c|c|c|}
\hline 15 & Orthetrum glaucum (Brauer, 1865) & & & $\mathrm{X}$ & & & \\
\hline 16 & Orthetrum luzonicum (Brauer, 1868) & & & & $\mathrm{X}$ & & $\mathrm{X}$ \\
\hline 17 & $\begin{array}{l}\text { Orthetrum pruinosum (Burmeister, } \\
1839 \text { ) }\end{array}$ & $\mathrm{X}$ & $\mathrm{X}$ & & $\mathrm{X}$ & $\mathrm{X}$ & $\mathrm{X}$ \\
\hline 18 & Orthetrum taeniolatum (Schneider,1845) & & $\mathrm{X}$ & $\mathrm{X}$ & $\mathrm{X}$ & $\mathrm{X}$ & $\mathrm{X}$ \\
\hline 19 & Pantala flavescens (Fabricius, 1798) & $\mathrm{X}$ & & $\mathrm{X}$ & $\mathrm{X}$ & & $\mathrm{X}$ \\
\hline 20 & Potamarcha congener* (Rambur, 1842) & & & & & $\mathrm{X}$ & \\
\hline 21 & Rhyothemis variegata (Linnaeus, 1763) & $\mathrm{X}$ & & & $\mathrm{X}$ & & $\mathrm{X}$ \\
\hline 22 & Tholymis tillarga (Fabricius, 1798) & $\mathrm{X}$ & & & & & \\
\hline 23 & Tramea limbata (Desjardins, 1832) & $\mathrm{X}$ & & & & & $\mathrm{X}$ \\
\hline 24 & Trithemis aurora (Burmeister, 1839) & $\mathrm{X}$ & $\mathrm{X}$ & $\mathrm{X}$ & & $\mathrm{X}$ & $\mathrm{X}$ \\
\hline 25 & Trithemis festiva (Rambur, 1842) & $\mathrm{X}$ & & $\mathrm{X}$ & & $\mathrm{X}$ & $\mathrm{X}$ \\
\hline 26 & Trithemis pallidinervis (Kirby, 1889) & $\mathrm{X}$ & & & $\mathrm{X}$ & & \\
\hline 27 & Urothemis signata Rambur, 1842 & $\mathrm{X}$ & $\mathrm{X}$ & & & & $\mathrm{X}$ \\
\hline \multicolumn{8}{|c|}{ Family: Macromiidae (1) } \\
\hline 28 & Epophthalmia vittata Burmeister,1839 & & $\mathrm{X}$ & & & & $\mathrm{X}$ \\
\hline \multicolumn{8}{|c|}{$\begin{array}{l}\text { Suborder: Zygoptera } \\
\text { Family: Chlorochyphidae (2) }\end{array}$} \\
\hline 29 & Libellago lineata* $($ Burmeister, 1839) & $\mathrm{X}$ & & & & $\mathrm{X}$ & \\
\hline 30 & $\begin{array}{l}\text { Heliocypha bisignata (Hagen in Selys, } \\
1853 \text { ) }\end{array}$ & & & & & $\mathrm{X}$ & $\mathrm{X}$ \\
\hline \multicolumn{8}{|c|}{ Family: Coenagrionidae (11) } \\
\hline 31 & Agriocnemis pygmaea (Rambur, 1842) & $\mathrm{X}$ & $\mathrm{X}$ & & $\mathrm{X}$ & $\mathrm{X}$ & $\mathrm{X}$ \\
\hline 32 & Cercion dyeri Fraser, 1920 & $\mathrm{X}$ & & & & & \\
\hline 33 & $\begin{array}{l}\text { Ceriagrion coromandelianum (Fabricius, } \\
\text { 1798) }\end{array}$ & $\mathrm{X}$ & $\mathrm{X}$ & & $\mathrm{X}$ & & $\mathrm{X}$ \\
\hline 34 & Ischnura aurora (Brauer, 1865) & $\mathrm{X}$ & $\mathrm{X}$ & & & & $\mathrm{X}$ \\
\hline 41 & Ischnura nursei* (Morton,1907) & & $\mathrm{X}$ & & & & \\
\hline 35 & Ischnura senegalensis (Rambur, 1842) & $\mathrm{X}$ & $\mathrm{X}$ & & $\mathrm{X}$ & & $\mathrm{X}$ \\
\hline 36 & Pseudagrion spp. & & & & $\mathrm{X}$ & & \\
\hline 37 & Pseudagrion decorum (Rambur, 1842) & $\mathrm{X}$ & $\mathrm{X}$ & & $\mathrm{X}$ & $\mathrm{X}$ & \\
\hline 38 & Pseudagrion hypermelas (Selys, 1876 ) & $\mathrm{X}$ & & & & & \\
\hline 39 & $\begin{array}{l}\text { Pseudagrion microcephalum (Rambur, } \\
1842 \text { ) }\end{array}$ & & $\mathrm{X}$ & & & & \\
\hline 40 & Pseudagrion rubriceps (Selys, 1876b) & $\mathrm{X}$ & $\mathrm{X}$ & $\mathrm{X}$ & $\mathrm{X}$ & $\mathrm{X}$ & $\mathrm{X}$ \\
\hline \multicolumn{8}{|c|}{ Family: Platycnemididae (2) } \\
\hline 42 & Copera marginipes (Rambur, 1842) & $\mathrm{X}$ & & & & & $\mathrm{X}$ \\
\hline 43 & $\begin{array}{l}\text { Disparoneura quadrimaculata } \\
\text { (Rambur,1842) }\end{array}$ & $\mathrm{X}$ & & $\mathrm{X}$ & & $\mathrm{X}$ & \\
\hline \multicolumn{8}{|c|}{ Family: Calopterygidae (1) } \\
\hline 44 & Vestalis gracilis (Rambur, 1842) & & & & & $\mathrm{X}$ & \\
\hline
\end{tabular}


Appendix S2. Distribution of odonates across seasons. Abbreviation and symbol: X, presence.

\begin{tabular}{|c|c|c|c|}
\hline & Pre-monsoon & Monsoon & Post-monsoon \\
\hline \multicolumn{4}{|c|}{ Suborder: Anisoptera (Dragonfly) } \\
\hline Acisoma panorpoides & & $\mathrm{X}$ & $\mathrm{X}$ \\
\hline Anax guttatus & $\mathrm{X}$ & $\mathrm{X}$ & \\
\hline Anax immaculifrons & $\mathrm{X}$ & & \\
\hline Anax spp. & $\mathrm{X}$ & & \\
\hline Brachythemis contaminata & $\mathrm{X}$ & $\mathrm{X}$ & $\mathrm{X}$ \\
\hline Bradinopyga geminata & $\mathrm{X}$ & $\mathrm{X}$ & \\
\hline Burmagomphus spp. & & $\mathrm{X}$ & \\
\hline Crocothemis servilia & $\mathrm{X}$ & $\mathrm{X}$ & $\mathrm{X}$ \\
\hline Diplacodes trivialis & $\mathrm{X}$ & $\mathrm{X}$ & $\mathrm{X}$ \\
\hline Epophthalmia vittata & $\mathrm{X}$ & & $\mathrm{X}$ \\
\hline Ictinogomphus rapax & $\mathrm{X}$ & $\mathrm{X}$ & $\mathrm{X}$ \\
\hline Neurothemis fulvia & & $\mathrm{X}$ & \\
\hline Orthetrum glaucum & $\mathrm{X}$ & & \\
\hline Orthetrum luzonicum & & $\mathrm{X}$ & \\
\hline Orthetrum pruinosum & $\mathrm{X}$ & $\mathrm{X}$ & $\mathrm{X}$ \\
\hline Orthetrum taeniolatum & $\mathrm{X}$ & $\mathrm{X}$ & $\mathrm{X}$ \\
\hline Orthretum sabina & $\mathrm{X}$ & $\mathrm{X}$ & $\mathrm{X}$ \\
\hline Pantala flavescens & $\mathrm{X}$ & $\mathrm{X}$ & $\mathrm{X}$ \\
\hline Rhyothemis variegata & $\mathrm{X}$ & $\mathrm{X}$ & \\
\hline Tholymis tillarga & $\mathrm{X}$ & & \\
\hline Tramea limbata & $\mathrm{X}$ & $\mathrm{X}$ & \\
\hline Trithemis aurora & $\mathrm{X}$ & $\mathrm{X}$ & $\mathrm{X}$ \\
\hline Trithemis festiva & $\mathrm{X}$ & $\mathrm{X}$ & $\mathrm{X}$ \\
\hline Trithemis pallidinervis & $\mathrm{X}$ & $\mathrm{X}$ & $\mathrm{X}$ \\
\hline Urothemis signata & $\mathrm{X}$ & $\mathrm{X}$ & \\
\hline \multicolumn{4}{|c|}{ Suborder: Zygoptera (Damselfly) } \\
\hline Agriocnemis pygmaea & $\mathrm{X}$ & $\mathrm{X}$ & $\mathrm{X}$ \\
\hline Cercion dyeri & & $\mathrm{X}$ & \\
\hline Ceriagrion coromandelinum & $\mathrm{X}$ & $\mathrm{X}$ & $\mathrm{X}$ \\
\hline Copera marginipes & $\mathrm{X}$ & $\mathrm{X}$ & $\mathrm{X}$ \\
\hline Disparoneura quadrimaculata & $\mathrm{X}$ & $\mathrm{X}$ & \\
\hline Heliocypha bisignata & & $\mathrm{X}$ & $\mathrm{X}$ \\
\hline Ischnura aurora & & $\mathrm{X}$ & $\mathrm{X}$ \\
\hline Ischnura senegalensis & $\mathrm{X}$ & $\mathrm{X}$ & $\mathrm{X}$ \\
\hline Pseudagrion decorum & $\mathrm{X}$ & $\mathrm{X}$ & $\mathrm{X}$ \\
\hline Pseudagrion hypermelas & & $\mathrm{X}$ & $\mathrm{X}$ \\
\hline Pseudagrion microcephalum & & & $\mathrm{X}$ \\
\hline Pseudagrion rubriceps & $\mathrm{X}$ & $\mathrm{X}$ & $\mathrm{X}$ \\
\hline Pseudagrion spp. & & $\mathrm{X}$ & \\
\hline Vestalis apicalis & & $\mathrm{X}$ & \\
\hline
\end{tabular}

